\title{
Formation and propagation of solitonlike defect clusters in confined active nematics with chiral anchoring
}

\author{
Zhong-Yi Li, De-Qing Zhang, and Bo Li ${ }^{*} *$ \\ Institute of Biomechanics and Medical Engineering, Applied Mechanics Laboratory, \\ Department of Engineering Mechanics, Tsinghua University, Beijing 100084, China
}

(Received 15 February 2021; accepted 15 June 2021; published 28 June 2021)

\begin{abstract}
Understanding the emergent nonequilibrium dynamics of collective units is crucial for steering and engineering active many-body systems. Here, we explore flow transitions and topological defect dynamics of channel-confined active nematics under chiral boundary anchoring. We discover that the anchoring chirality can engender unanticipated solitonlike defect clusters, where activity-excited topological defects self-localize into small cohesive clusters that unidirectionally propagate as dynamically stable solitons. Defects continuously move, nucleate, and annihilate locally, which causes periodic cluster reconfiguration and energy oscillations, reflecting the structural degeneracy of defect clusters. The boundary anchoring chirality not only determines the direction of background active flows but also remarkably speeds them up, which underlies the emergence of solitonlike defect clusters. We further show that the periodicity and structural reconfiguration of defect clusters can be tuned using specified initial orientation fields. These findings could motivate direct programmatic control strategies for engineered active materials and provide insights into the collective behaviors of living organisms within confining tracks during life processes.
\end{abstract}

DOI: 10.1103/PhysRevResearch.3.023253

\section{INTRODUCTION}

Active many-body systems such as cell populations [1-3], cytoskeleton biopolymers [4-7], bacterial suspensions [8-12], animal collections [13-15], and robot swarms [16] ordinarily display diverse dynamics and patterns, including directional motion $[2,5,17]$, sustained rotation $[2,18]$, giant density fluctuations $[19,20]$, synchronized oscillations [10,21], mesoscale turbulence [8], and jamming transition [22,23]. These spectacular behaviors are closely relevant to life processes and engineering applications [2,24-26] and derive primarily from the specific activity powered by continuous energy injection and rich interactions between the units of systems [27-29]. Owing to their utmost importance for physics, materials science, biological processes, and engineering, nonequilibrium defect and flow dynamics emerging in active nematics, which are quintessential active systems with elongated units, have aroused surging interests [30-36]. It was found that environmental friction [37-40] and chirality of units [19,32,41,42] can screen hydrodynamic flows and regularize defect trajectories in active nematics.

Recent studies demonstrated that active nematic flows and topological defects are sensitive to the geometry of the space that they inhabit [43-45]. Under circular confinements of

\footnotetext{
*Corresponding author: libome@tsinghua.edu.cn

Published by the American Physical Society under the terms of the Creative Commons Attribution 4.0 International license. Further distribution of this work must maintain attribution to the author(s) and the published article's title, journal citation, and DOI.
}

appropriate diameters, topological defects annihilate pairwise until two remaining $+\frac{1}{2}$ ones, which can either reach a stable position or self-organize into persistent rotation, or even merge into one +1 defect ultimately $[39,46,47]$. By contrast, under either two- or three-dimensional channel confinements, active nematics may spontaneously generate defect-free directed flow, vortex or vortex-roller lattice, and mesoscale turbulence, depending on the channel dimensions [48-51]. Due to the effects of the dynamical interactions, adhesion, and anisotropic shapes of units, as well as wall entrapment and surface microstructures of confinements, many active nematics often exhibit boundary alignment with tilted angles [26,52-55], referred to as chiral anchoring, which may in turn influence the collective dynamics of systems, especially when the confinement dimension is comparable with the characteristic active length scales [56,57]. Despite the profound importance of boundary-anchoring chirality, how it controls flow and defect dynamics in the bulk of active nematics remains incompletely understood.

In this paper, we investigate the emerging dynamics of channel-confined active nematics under chiral boundary anchoring. We show that the anchoring chirality can give rise to spontaneous coherent background flows, whose direction and speed are well controlled by the tilted anchoring angle. The chiral anchoring can engender unexpected solitonlike defect clusters, which are locally organized by several topological defects and stably transported by directional background flows. Through tracking the energy of defect clusters, we clarify the degeneracy of multiple defect configurations and elucidate the emerging structural reconfiguration. The mechanism behind the formation and propagation of solitonlike defect clusters is deciphered. We further illustrate that using 
the initial orientation fields, the solitonlike defect clusters can be either synchronized in phase or desynchronized to antiphase.

\section{MODEL DESCRIPTION}

Our model is based on the active nematic theory [28,44]. It uses a traceless symmetric tensor $\mathbf{Q}$ to describe the orientation order of the active units $\mathbf{Q}=q(\mathbf{n n}-\mathbf{I} / 3)$, where $\mathbf{n}$ is the orientation field of the rod-shaped units and satisfies $\mathbf{n}=-\mathbf{n}, q$ denotes the order parameter describing the local order degree, and $\mathbf{I}$ is the unit tensor. The evolution of $\mathbf{Q}$ is controlled by the Beris-Edwards equation [58,59]:

$$
\left(\partial_{t}+\mathbf{v} \cdot \nabla\right) \mathbf{Q}-\mathbf{S}-\Gamma \mathbf{H}=0,
$$

where $\quad \mathbf{S}=(\lambda \mathbf{E}+\boldsymbol{\Omega})(\mathbf{Q}+\mathbf{I} / 3)+(\mathbf{Q}+\mathbf{I} / 3)(\lambda \mathbf{E}-\boldsymbol{\Omega})-$ $2 \lambda(\mathbf{Q}+\mathbf{I} / 3) \operatorname{tr}(\mathbf{Q W})$ is the corotation term which determines the alignment of rod-shaped units in response to gradients in the velocity field $\mathbf{v}, \mathbf{W}=\nabla \mathbf{v}$, and $\mathbf{I}$ is the unit tensor. Here, $\mathbf{E}$ and $\boldsymbol{\Omega}$ denote the symmetrical and antisymmetric parts of $\mathbf{W}$, respectively. Also, $\lambda$ is the alignment coefficient which controls the relative dominance of $\mathbf{E}$ and $\boldsymbol{\Omega}$, which account for the flow aligning and flow tumbling of units, respectively. Additionally, $\Gamma$ is the rotational diffusivity, and $\mathbf{H}$ is the molecular field determined by the Landau-de Gennes free energy $F_{\mathrm{b}}$ in the bulk [44]

$$
\mathbf{H}=-\frac{\delta F_{\mathrm{b}}}{\delta \mathbf{Q}}+\frac{\mathbf{I}}{3} \operatorname{tr}\left(\frac{\delta F_{\mathrm{b}}}{\delta \mathbf{Q}}\right) .
$$

The Landau-de Gennes free energy $F_{\mathrm{b}}$ can be expressed as

$$
\begin{aligned}
F_{\mathrm{b}}= & \int_{V}\left\{\frac{A_{0}(1-\gamma / 3)}{2} \operatorname{tr}\left(\mathbf{Q}^{2}\right)-\frac{A_{0} \gamma}{3} \operatorname{tr}\left(\mathbf{Q}^{3}\right)\right. \\
& \left.+\frac{A_{0} \gamma}{4}\left[\operatorname{tr}\left(\mathbf{Q}^{2}\right)\right]^{2}+\frac{1}{2} K(\nabla \mathbf{Q})^{2}\right\} d V,
\end{aligned}
$$

where $A_{0}$ and $\gamma$ are parameters that can be used to tune the nematic phase behaviors. The order parameter $q$ of $\mathbf{Q}$ in the uniform state satisfies $q=\frac{1}{4}+\frac{3}{4} \sqrt{1-\frac{8}{3 \gamma}} ; K$ is the Frank elastic constant describing the orientational stiffness. The evolution of the $\mathbf{Q}$ field at the boundary is controlled by the anchoring energy $F_{\text {an }}$ and satisfies [60-62]

$$
\partial_{t} \mathbf{Q}=\Gamma \mathbf{H}_{\mathrm{s}},
$$

where $\mathbf{H}_{\mathrm{s}}$ stands for the molecular field of boundary and is determined by

$$
\mathbf{H}_{\mathrm{s}}=K \boldsymbol{v} \cdot \nabla \mathbf{Q}-\frac{\delta F_{\mathrm{an}}}{\delta \mathbf{Q}},
$$

with $v$ being the normal direction of the boundary. The orientation of the units at the boundary walls is prescribed by introducing boundary-anchoring energy [63]:

$$
F_{\text {an }}=\int_{S} \frac{W}{2}\left(\mathbf{Q}-\mathbf{Q}_{\text {an }}\right)^{2} d S,
$$

where $W$ is the anchoring strength, and $\mathbf{Q}_{\mathrm{an}}=q\left(\mathbf{n}_{\mathrm{an}} \mathbf{n}_{\mathrm{an}}-\right.$ $\mathbf{I} / 3$ ), with $\mathbf{n}_{\text {an }}$ being the preferred orientation of units at the boundaries. We consider a straight channel, whose centerline coincides with the $x$ axis. Generally, the anchoring angles at the top and bottom boundaries $\left(\theta_{\mathrm{an}}^{\mathrm{t}}\right.$ and $\left.\theta_{\mathrm{an}}^{\mathrm{b}}\right)$ are adjustable and can be either symmetric $\left(\theta_{\mathrm{an}}^{\mathrm{t}}=-\theta_{\mathrm{an}}^{\mathrm{b}}\right)$ or antisymmetric $\left(\theta_{\mathrm{an}}^{\mathrm{t}}=\theta_{\mathrm{an}}^{\mathrm{b}}\right)$. A tilted angle, neither parallel nor perpendicular to the channel walls, will cause the difference between the left and right tilt of the boundaries and destroy the spatial reflection symmetry; thus, it is called chiral boundary anchoring.

The velocity field $\mathbf{v}$ is governed by the incompressible condition $\nabla \cdot \mathbf{v}=\mathbf{0}$ and the Navier-Stokes equation [60,62,64]:

$$
\rho\left(\partial_{t}+\mathbf{v} \cdot \nabla\right) \mathbf{v}=\nabla \cdot\left(\sigma_{\mathrm{p}}+\sigma_{\text {act }}\right),
$$

where $\rho$ is the density of the active nematic and assumed to be constant; $\sigma_{\mathrm{p}}$ denotes the passive stress, which is given as $\sigma_{\mathrm{p}}=-\lambda \mathbf{H}(\mathbf{Q}+\mathbf{I} / 3)-\lambda(\mathbf{Q}+\mathbf{I} / 3) \mathbf{H}+$ $2 \lambda(\mathbf{Q}+\mathbf{I} / 3) \operatorname{tr} \mathbf{Q H}+\mathbf{Q H}-\mathbf{H Q}-\nabla \mathbf{Q}: \frac{\delta F_{\mathrm{b}}}{\delta \nabla \mathbf{Q}}+2 \eta \mathbf{E}-p \mathbf{I} ; \eta$ is the isotropic viscosity, and $p$ is the hydrodynamic pressure [44,62]. Here, $\sigma_{\text {act }}=-\zeta \mathbf{Q}$ denotes the active stress, with $\zeta$ being the activity coefficient, which describes the spontaneous extension $(\zeta>0)$ or contraction $(\zeta<0)$ of the unit $[65,66]$.

We adopt the hybrid lattice Boltzmann method to numerically solve Eqs. (1) and (7) [63]. Our simulation areas are rectangular stripelike windows with grids of $300 \times 30$, $(150,500,800,1000) \times 30$, and $300 \times(15,25,35,45)$, unless stated otherwise. The no-slip boundary of the velocity field is realized by the bounce-back rule, and the preferred boundary orientation is controlled by anchoring angles at the upper and lower boundaries of the channel. Periodic boundary conditions are used for the left and right ends. The initial orientation is randomly distributed, and the velocity field is 0 . We use the simulated space and time steps as the space and time units for normalization. The typical parameter values are taken as $A_{0}=0.1, \gamma=3, K=0.04, \lambda=0.9, \eta=2 / 3, \zeta=$ $0.02, \rho=1, \Gamma=0.34, W=0.1$, and $\theta_{\text {an }}^{\mathrm{t}}=-\theta_{\text {an }}^{\mathrm{b}}=-\pi / 4$, unless stated otherwise.

\section{RESULTS}

Chiral anchoring may lead to spontaneous directed flows for symmetric anchoring, a nonequilibrium steady state with driven energy from active stress [55,67]. We next focus on active nematics with symmetric boundary anchoring. To gain theoretical insights, we consider a simplified, onedimensional model system, where any gradients are allowed only in the $y$ direction of channel width [68]. From Eq. (1), we obtain the orientation angle $\theta$ (see Appendix A):

$$
\partial_{y}{ }^{2} \theta=\frac{\zeta}{8 \eta \Gamma K} \sin 2 \theta-\frac{\zeta \lambda}{12 \eta \Gamma K} \sin 4 \theta .
$$

The last item in Eq. (8) reflects the effect of flow alignment or flow tumbling. Generally, flow alignment causes a higher flow speed than flow tumbling (see Appendix A). The boundary condition corresponding to symmetric anchoring is set to be $\theta(y= \pm H / 2)=\mp \theta_{\text {an }}$, where $H$ is the channel width, and $0^{\circ} \leqslant \theta_{\text {an }} \leqslant 90^{\circ}$. Solving Eq. (8) under such a boundary condition results in an odd function $\theta$ with respect to $y$, in which the unit orientation extends along the $x$ axis at the center of the channel. From Eq. (7), we obtain the flow velocity $v_{x}$ along the $y$ direction:

$$
v_{x}=\int_{-H / 2}^{y} \frac{\zeta}{4 \eta} \sin 2 \theta d y .
$$


Equation (9) indicates that the flow velocity $v_{x}>0$ anywhere and takes on a symmetric distribution about the $x$ axis, where $v_{x}$ attains the maximum at $y=0$, as shown in Appendix A. In this steady state, the director field adopts a "splay" configuration, which is guaranteed by the flow alignment. The flow direction can be easily reversed when we set the chiral anchoring as $\theta(y= \pm H / 2)= \pm \theta_{\mathrm{an}}$. It suggests that the flow direction of the active nematics in the steady state can be well controlled by the boundary-anchoring chirality, distinct from the active laminar flows induced by spontaneous symmetry breaking under achiral boundary anchoring, whose left-right flow direction is virtually random [49-51,69]. Controlling the flow direction can realize the directional transport of active fluid, which provides a potential design strategy for active microfluidic chips. Moreover, channel-confined active nematics under symmetric chiral boundary anchoring may confer a similar stable self-propulsion velocity to active polar fluids, resulting in the absence of Galileo invariance $[67,70]$. It implies that these confined active nematics, which behave like active polar fluids [71-73], can transmit topological sound modes in the nonequilibrium steady state.

To grasp a whole picture of nonequilibrium dynamics, we perform numerical simulations of the channel-confined active nematics by using the hybrid lattice Boltzmann algorithm. We show that the emerging dynamics and self-organization of the confined active nematics are dictated by the anchoring angle $\left(\theta_{\mathrm{an}}\right)$ at the boundary walls and the activity number $\alpha=H \sqrt{\zeta / K}$, which weighs the effects of the unit activity and the geometric screening [43]. We establish a phase diagram through $\theta_{\text {an }}$ and $\alpha$, and herein, four regimes (I-IV) can be distinguished generally [Fig. 1(a)]. When $\alpha$ is low, the system prefers a steady unidirectional flow state independent of $\theta_{\text {an }}$, where topological defects disappear (regime I). The units adopt a splay configuration at the center of the channel, and the left-right direction of active flow is controlled by the anchoring angle [Fig. 1(b)], in line with our theoretical prediction from the one-dimensional model [Eq. (9)].

Moderately increasing activity number will destabilize the steady state in regime I, rendering topological defects that move regularly. In this scenario, two regimes (II and III) can be distinguished according to defect motion [Fig. 1(a)]. In regime II, the topological defects spontaneously organize into an ordered structure [Fig. 1(c)], leading to a defect dancing state and vortex lattice, as reported previously $[37,43,49]$. Intriguingly, in some ranges of anchoring angles (regime III), the topological defects can self-organize into cohesive clusters, each of which localizes within a narrow region and is periodically segregated far from neighboring clusters [Fig. 1(d)]. Each cluster contains one or two pairs of $\pm \frac{1}{2}$ defects, which move, nucleate, and annihilate locally, but simultaneously propagate together as a soliton along the channel. Evidently, the emerging solitonlike defect clusters break the spatial translation symmetry of the system and engender a net flow remarkably faster than in regime II. Further increasing activity number may destroy the regular defect motion, yielding confined mesoscale active turbulence (regime IV) [Fig. 1(e)], which was also observed in channel-confined active nematics with achiral anchoring [48]. For antisymmetric boundaries, confined shear flow will be observed (see
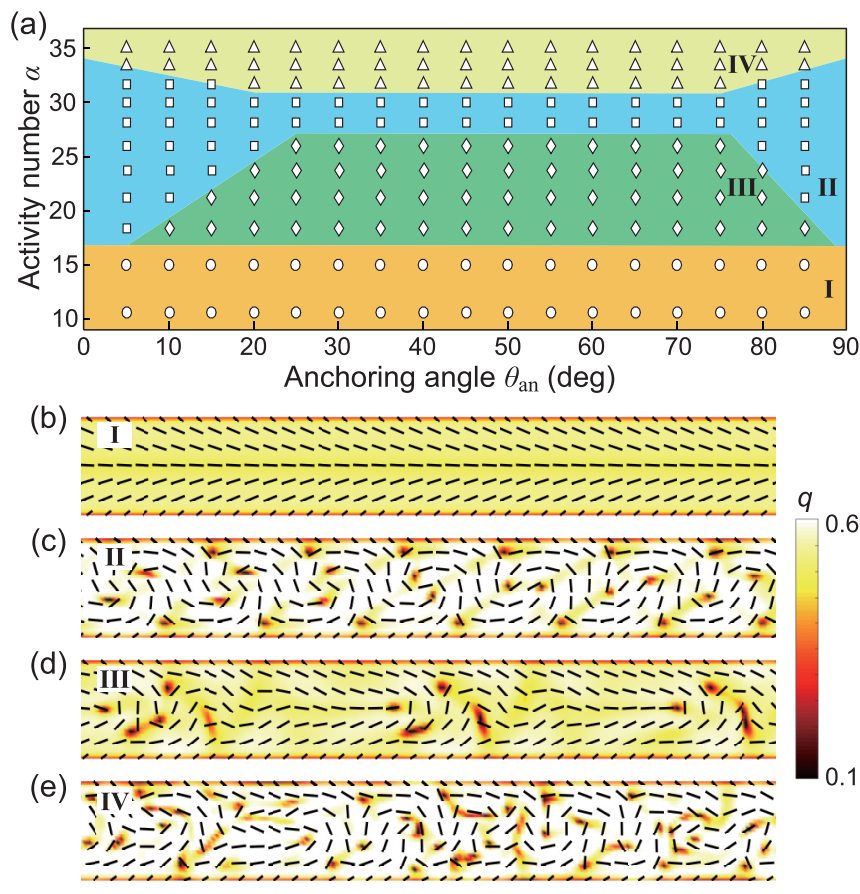

FIG. 1. (a) Phase diagram of channel-confined active nematics with symmetric chiral anchoring at the boundary walls. (b) Unidirectional laminar flow (regime I). (c) Defect dancing (regime II). (d) Solitonlike defect cluster (regime III). (e) Mesoscale active turbulence (regime IV).

Appendix B for details). This nonequilibrium dynamics has been found in animal cells within confining stripes [52].

We next concentrate on regime III. Outside the narrow regions where solitonlike defect clusters localize, the active flow is almost laminar and has only the $x$ direction velocity component [Fig. 2(a) and 2(b)]. We calculate the Landaude Gennes energy of the system, and it peaks exactly at the location of defect clusters as expected [Fig. 2(c)]. The emergence of solitonlike defect clusters with high energy can be attributed to the self-localization of topological defects, who individually have high energy. Additionally, the energy distribution along the $x$ direction exhibits periodic nonlinearity, and the waveform of clusters remains nearly permanent with time evolution, verifying the balance between dispersion and nonlinearity, a common hallmark of solitons [74]. The energy dissipation induced by viscosity is supplemented by the sustained energy injection, which maintains and powers the stable propagation of solitonlike defect clusters. Likewise, we examine the kinetic energy of the active system and find it peaks at the location of self-propelled $+\frac{1}{2}$ defects [Fig. 2(d)]. The spatial localization of defects and their nondissipation of energy suggest that the solitonlike defect clusters confer the characteristics of a "quasiparticle" [74]. The quasiparticle nature of multidefects has been observed in bound $+\frac{1}{2}$ defect pairs, whose structures maintain stably in the chaotic flow, revealing the topological protection mechanism of defect structures [75].

During the propagation, the solitonlike defect clusters undergo periodical structure reconfiguration through local motion, nucleation, and annihilation of $\pm \frac{1}{2}$ defects. We next 
(a)
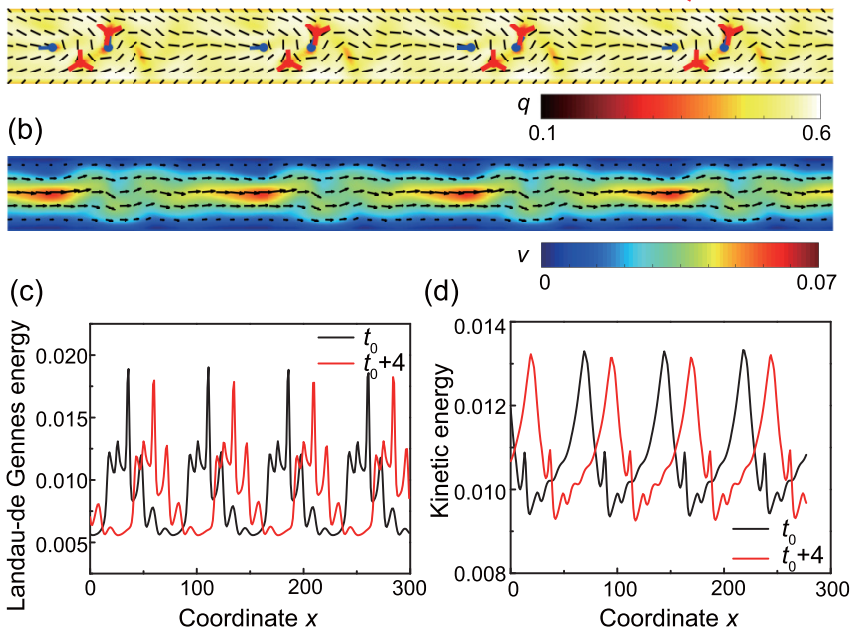

FIG. 2. Simulation results for orientation order $\mathbf{Q}$ and velocity $\mathbf{v}$. (a) $\mathbf{Q}$ field. Active units are indicated by black line segments. (b) $\mathbf{v}$ field. Black arrows denote the direction of velocity. (c) Distribution of Landau-de Gennes energy at time $t_{0}$ and $t_{0}+4$. (d) Distribution of kinetic energy at time $t_{0}$ and $t_{0}+4$. Here, $\alpha=21.2$, and $\theta_{\text {an }}^{\mathrm{t}}=$ $-\theta_{\mathrm{an}}^{\mathrm{b}}=-\pi / 4$.

take a defect cluster as an example to probe this process [Fig. 3(a)]. In state A, there exists only a pair of $\pm \frac{1}{2}$ defects, which tend to move together. The rightward motion of the inertial $-\frac{1}{2}$ defect, driven by the background flow, contributes to escaping from the approaching of the nearby $+\frac{1}{2}$ defect and, thus retarding the annihilation of oppositely charged defects. Therefore, state A can be dynamically maintained up to the formation of a new pair of $\pm \frac{1}{2}$ defects at the bottom right (state $\mathrm{B}$ ). In addition to the overall rightward translation, the new $+\frac{1}{2}$ defect in state $B$ also moves upward and

(a)

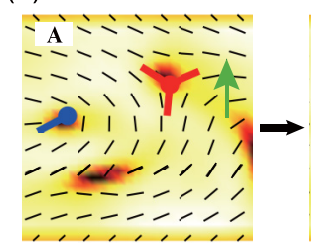

(b)

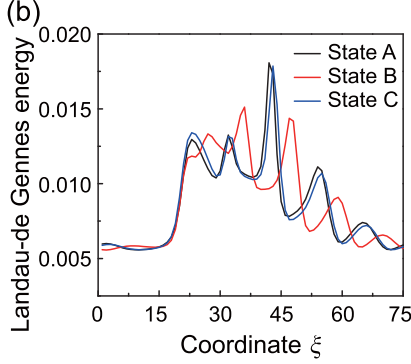

(c)

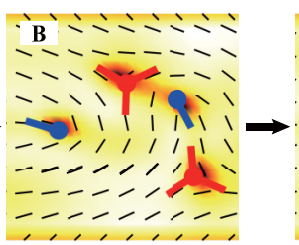

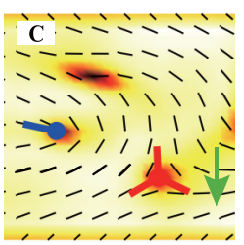
$\therefore=$

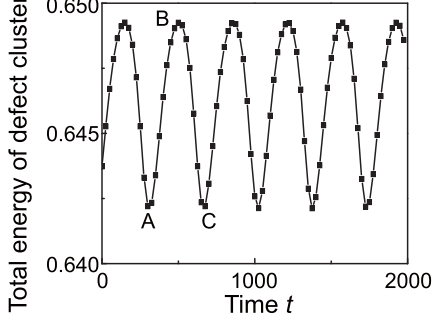

FIG. 3. (a) Snapshots of topological reconfiguration of a solitonlike defect cluster in different states. Green arrows indicate the mirror symmetry between states A and C. (b) Spatial distribution of Landau-de Gennes energy $E(\xi, t)$ in states A, B, and C corresponding to (a). (c) Evolution of total energy in a solitonlike defect cluster over time. annihilates with the original $-\frac{1}{2}$ defect after they meet. Finally, the solitonlike defect cluster reinstates the configuration containing a pair of $\pm \frac{1}{2}$ defects only (state C). Although state $\mathrm{C}$ seems to be markedly different from state $\mathrm{A}$, they are mirror symmetric with respect to the central axis of the channel. Similarly, state $\mathrm{C}$ can transition to state A by creating a new defect pair and subsequent annihilation, thereby attaining continuous, periodical reconfiguration of the solitonlike structure.

To decipher the topological reconfiguration of solitonlike defect clusters, we introduce the Galileo transformation $\xi=$ $x-V_{0} t$ on the space coordinate $x$, where $V_{0}$ is the propagation speed of the defect clusters. After the Galileo transformation, the distribution of the Landau-de Gennes energy $E(\xi, t)$ is dynamically stable and displays small fluctuations during topological reconfiguration, as shown in Fig. 3(b). The configurations at states $\mathrm{A}$ and $\mathrm{C}$ have the same energy, confirming their mirror symmetric identity. We further calculate the total energy of a cluster over time and find it exhibits regular oscillation [Fig. 3(c)]. During the transition from state A to C, the total energy first rises and then falls, reaching the maximum at state B. Evidently, the mirror symmetric states A and C are the two simplest configurations that not only feature vanishing total topological charges but also have the same lowest energy, suggesting that they are different degenerate states. The switch back and forth between these two degenerate states is reminiscent of the bistable states and their mutual transformation in structural phase transitions [76,77].

We now examine the mechanism underlying the emergence and stable propagation of solitonlike defect clusters. We average the flow velocity over space and time, denoted by $\left\langle v_{x}\right\rangle$, to quantify the flow capacity of directional flow driven by chiral boundary anchoring. Figure $4(\mathrm{a})$ demonstrates that $\left\langle v_{x}\right\rangle$ is positively correlated to the anchoring angle $\theta_{\text {an }}$ with relatively low activity $[\alpha=15$, regime I in Fig. 1(a)]. With high activity $\left[\alpha=30\right.$, regime II in Fig. 1(a) ], $\left\langle v_{x}\right\rangle$ vanishes when $\theta_{\text {an }}=0^{\circ}$ or $90^{\circ}$ and reaches the maximum around $\theta_{\text {an }}=65^{\circ}$ under given parameters. This suggests that the tilted anchoring at the boundary may tame nematics, particularly with high activity, and remarkably speed them up, inducing a fast and directional background flow. Remarkably, the chiral anchoring angle can trigger the transition from defect dancing (regime II) to solitonlike defect cluster propagation (regime III), during which the average flow velocity is sharply enhanced [Fig. 4(b)]. There exist two critical anchoring angles in this transition. Beyond the lower threshold, the fast enough background flow can disrupt the defect dance, rendering solitonlike defect clusters that strikingly intensify the spatial translation symmetry breaking of the system; beyond the higher threshold, the localized solitonlike defect clusters restore to the evenly distributed defect dancing.

As shown in Fig. 4(c), when anchoring angle is small, self-propelled $+\frac{1}{2}$ defects often move much faster than passive $-\frac{1}{2}$ defects, which would facilitate the combinations of oppositely charged defects and thus defect annihilation. However, when the anchoring angle exceeds the lower threshold, the directional background flow is sharply accelerated, which quickens $\pm \frac{1}{2}$ defects but induces more velocity increment in passive $-\frac{1}{2}$ defects [Fig. 4(c)]. This behavior slows down the 
(a)

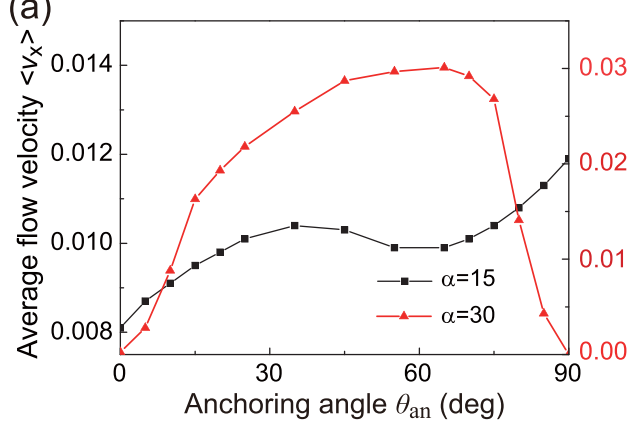

(b)

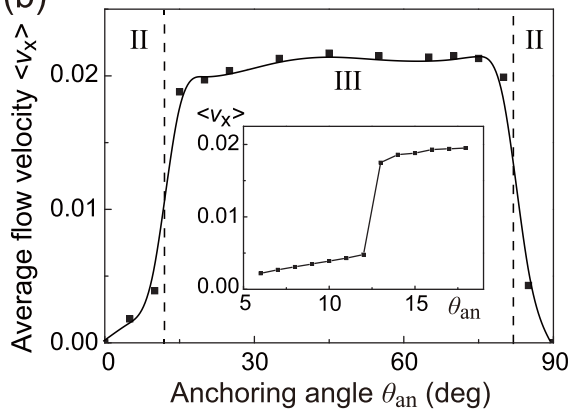

(c)

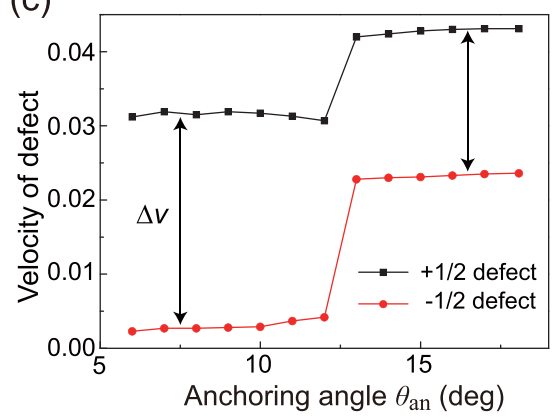

FIG. 4. (a) Variations of average flow velocity $\left\langle v_{x}\right\rangle$ vs anchoring angle $\theta_{\text {an }}$ under low and high activity numbers. (b) Variations of $\left\langle v_{x}\right\rangle$ vs $\theta_{\text {an }}$ under moderate activity number $(\alpha=21.2)$. In this situation, phase transition from defect dancing (regime II) to solitonlike defect clusters (regime III) can take place. (c) The motility discrepancy between $+\frac{1}{2}$ and $-\frac{1}{2}$ defects. $\Delta v$ is the velocity difference between $\pm \frac{1}{2}$ defects and significantly reduces when $\theta_{\text {an }}$ exceeds the lower threshold.

annihilation of topological defects and thereby enables longterm directional motion of $+\frac{1}{2}$ defects, which is indispensable to the generation and stable propagation of solitonlike defect clusters.

The balance between nonlinearity and dispersion of solitonlike defect clusters allows us to modulate the number of solitonlike defect clusters and the degeneracy or identity of adjacent defect clusters through initial orientation fields. We introduce an initial orientation $\theta_{0}=\Delta \theta \sin \left(\frac{2 \pi k}{L} x\right)$, where $L$ is the channel length and $k$ is the modulation parameter that has to be an integer. In this case, $2 k$ solitonlike defect clusters emerge in the channel, where every two adjacent clusters are desynchronized to antiphase (Fig. 5), with different degenerate states akin to states A and C shown in Fig. 3(a). This is because each peak or trough in the initial orientation can induce a degenerate state. As another example, we introduce an initial orientation $\theta_{0}=\Delta \theta\left|\sin \left(\frac{2 \pi k^{\prime}}{L} x\right)\right|$, where the modula-

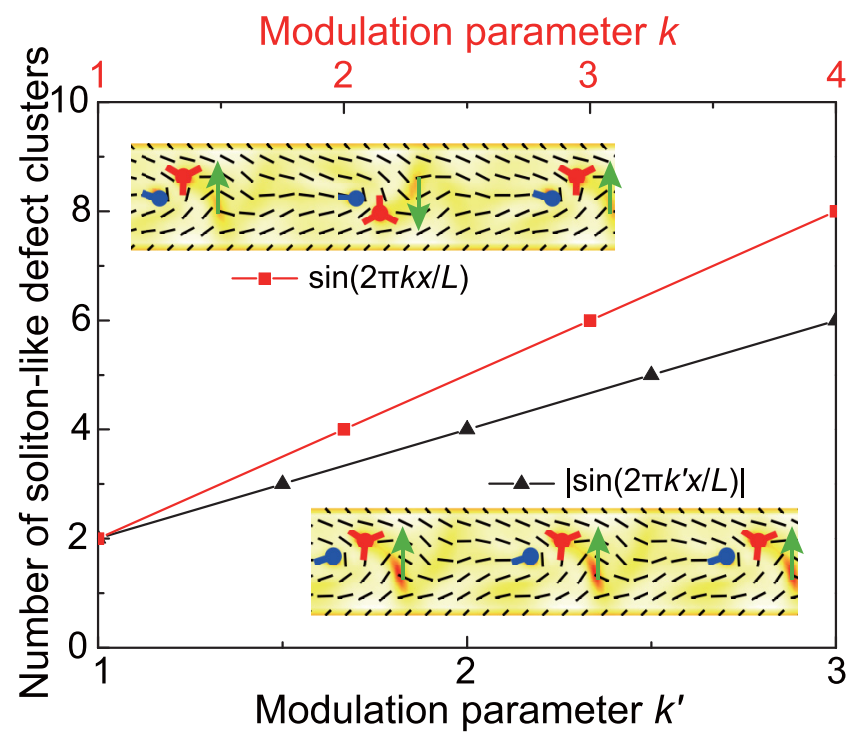

FIG. 5. Modulation of solitonlike defect clusters by initial orientation fields. In simulations corresponding to the insert snapshots, we take $k=3$ and $k^{\prime}=3$. Green arrows indicate different configurations of solitonlike defect clusters. tion parameter $k^{\prime}$ is set to be either an integer or half integer. It gives rise to $2 k^{\prime}$ solitonlike defect clusters in the channel, where all clusters are synchronized in phase and undergo the same structure reconfiguration (Fig. 5), attributable to all clusters being induced by the peaks in the initial orientation. These results demonstrate that the emerging solitonlike defect clusters controlled by chiral boundary anchoring can be further programed through modulation parameters, inspiring the possibility to develop soliton communication in active systems like that achieved in optical fibers [78].

\section{CONCLUSIONS}

In summary, we discover a solitonlike defect cluster state in channel-confined active nematics under chiral boundary anchoring. We elucidate the mechanisms underlying the formation of localized solitonlike defect clusters and show that their stable propagation and robust structure reconfiguration are well controlled by the anchoring chirality. Our results illustrate that the balance between nonlinearity and dispersion of solitonlike defect clusters allows for further programing them through modulation parameters of specified initial orientation fields. These findings could motivate possible routes toward engineering microfluidic platforms for quickening active flows, exciting and steering solitons, and achieving soliton communication. Recent experiments evidenced that some animal cells collectively form tilted alignment at the boundary confinements, leading to spontaneous shear flows [52]. While a previous study provided deep insights into this emerging boundary chirality, we pursue unveiling the regulatory role of chiral boundary alignment of cells in their collective dynamics and offering potential strategies to realize unidirectional propagation of defect clusters, which may be relevant to topological Kelvin waves found in neural cells [53]. In addition, our results indicate that it is possible to tune local and reproducible structures in the engineering of functionalities in active fluids or biosystems, which opens the possibility to engineer platforms for the controlled patterning of tissues and design of out-of-equilibrium spatiotemporal behaviors with the goal of developing dynamic materials and systems [79-81]. 


\section{ACKNOWLEDGMENTS}

We thank Yafei Zhang for valuable and supportive discussions. Supports from National Natural Science Foundation of China (Grants No. 11922207, No. 11961131005, and No. 11921002) are acknowledged.

\section{APPENDIX A: THEORETICAL SOLUTION}

To gain theoretical insight into the channel-confined active nematics under chiral boundary anchoring, we simplify Eqs. (1) and (7). Assume the order parameter $q$ to be constant. Thus, the order tensor $\mathbf{Q}$ can be written as $(\mathbf{n n}-\mathbf{I} / 3) / 2$. We ignore the higher order terms in the tensor $\mathbf{S}$ and the molecular field $\mathbf{H}$ and obtain $\mathbf{S}=2 / 3 \lambda \mathbf{E}+\boldsymbol{\Omega} \mathbf{Q}-\mathbf{Q} \boldsymbol{\Omega}$ and $\mathbf{H}=K \nabla^{2} \mathbf{Q}$. With these simplifications, Eq. (1) reduces to a partial differential equation of orientation angle $\theta$

$$
\begin{aligned}
\partial_{t} \theta+v_{x} \partial_{x} \theta+v_{y} \partial_{y} \theta= & \frac{2}{3} \lambda\left(W_{x y}+W_{y x}\right) \cos 2 \theta \\
& -\frac{4}{3} \lambda W_{x x} \sin 2 \theta-\frac{1}{2}\left(W_{y x}-W_{x y}\right) \\
& +\Gamma K \nabla^{2} \theta
\end{aligned}
$$

where $W_{i j}$ are components of $\mathbf{W}$, with $i, j=x, y$. In Eq. (7), only the active stress, the viscous stress, and hydrostatic pressure are maintained, and therefore, Eq. (7) becomes

$$
\begin{aligned}
\rho\left(\partial_{t} v_{x}+v_{x} \partial_{x} v_{x}+v_{y} \partial_{y} v_{x}\right)= & -\frac{\zeta}{2}\left(-\sin 2 \theta \partial_{x} \theta+\cos 2 \theta \partial_{y} \theta\right) \\
& +\eta\left[2 \partial_{x} W_{x x}+\partial_{y}\left(W_{x y}+W_{y x}\right)\right] \\
& +\partial_{x} p, \\
\rho\left(\partial_{t} v_{y}+v_{x} \partial_{x} v_{y}+v_{y} \partial_{y} v_{y}\right)= & -\frac{\zeta}{2}\left(\cos 2 \theta \partial_{x} \theta+\sin 2 \theta \partial_{y} \theta\right) \\
& +\eta\left[2 \partial_{y} W_{y y}+\partial_{x}\left(W_{x y}+W_{y x}\right)\right] \\
& +\partial_{y} p .
\end{aligned}
$$

Following previous studies [55,68], we assume that all the variables are uniformly distributed along the $x$ direction and are functions only of $y$ in the channel width direction and time, and $v_{y}$ vanishes. Hence, Eqs. (A1) and (A2) are simplified as

$$
\begin{aligned}
\frac{2}{3} \lambda W_{y x} \cos 2 \theta-\frac{1}{2} W_{y x}+\Gamma K \partial_{y}^{2} \theta & =0, \\
-\frac{\zeta}{2} \cos 2 \theta \partial_{y} \theta+\eta \partial_{y} W_{y x} & =0 .
\end{aligned}
$$

Solving Eq. (A5), we obtain

$$
W_{y x}=\frac{\zeta}{4 \eta} \sin 2 \theta .
$$

Substituting Eq. (A6) into (A4) leads to Eq. (8).

From the boundary-anchoring energy Eq. (6), we can derive the boundary conditions $\theta(y= \pm H / 2)=\mp \theta_{\text {an }}$ for symmetric chiral anchoring $\left(\theta_{\text {an }}^{\mathrm{t}}=-\theta_{\text {an }}^{\mathrm{b}}\right)$. The numerical integration of Eq. (8) under these boundary conditions shows an odd function for the solution of $\theta$ [Fig. 6(a)]. Integrating Eq. (A6), we get Eq. (9), which attains the maximum at $\theta=0$, that is, $v_{x}(y=0)=\int_{-H / 2}^{0} \frac{\zeta}{4 \eta} \sin 2 \theta d y$, as shown in Fig. 6(b), indicating that an active flow toward the positive $x$ axis direction emerges. In addition, it is worth noting that
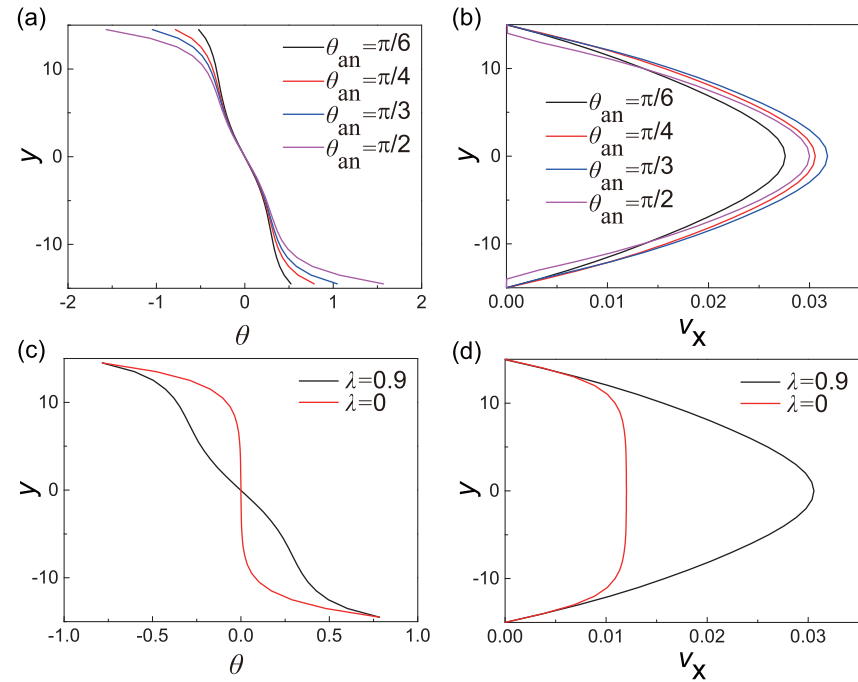

FIG. 6. Distributions of orientation angle $\theta$ and velocity $v_{x}$ with respect to $y$, which are obtained by numerical integration of Eqs. (8) and (9). The anchoring boundary conditions are $\theta(y= \pm H / 2)=$ $\mp \theta_{\text {an }}$, and no-slip velocity boundary is used. The activity coefficient $\zeta=0.01$. (a) and (b) Distributions of $\theta$ and $v_{x}$ with different anchoring angles. (c) and (d) Distributions of $\theta$ and $v_{x}$ with different alignment coefficients.

the last item in Eq. (8) reflects the effect of flow alignment or flow tumbling. Flow alignment can produce greater flow velocity than flow tumbling. The markedly sped-up flow provides a strong alignment effect to ensure that the orientation of the units at the center axis always keeps the $x$ direction, maintaining a splay configuration [Figs. 6(c) and $6(\mathrm{~d})]$.

\section{APPENDIX B: RESULT FOR ANTISYMMETRIC ANCHORING}

We provide the result for antisymmetric boundary anchoring with chirality, where we take $\theta_{\mathrm{an}}^{\mathrm{t}}=\theta_{\mathrm{an}}^{\mathrm{b}}=\pi / 4$. Our result shows that, at low activity, the antisymmetric chiral anchoring can induce stable shear flow with antiparallel velocity and (a)

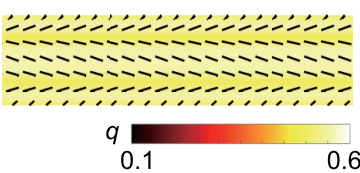

(c)

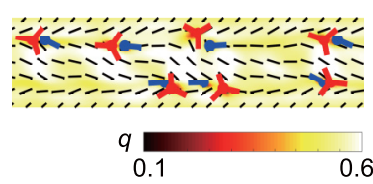

(b)

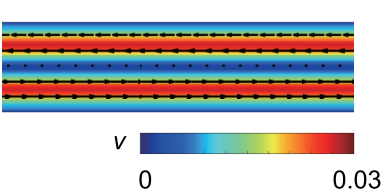

(d)

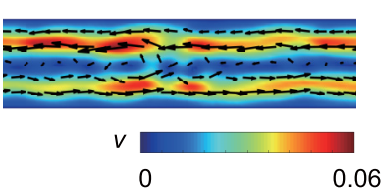

FIG. 7. Flow state and defect dynamics of active nematics under antisymmetric chiral anchoring, where we take $\left(\theta_{\mathrm{an}}^{\mathrm{t}}=\theta_{\mathrm{an}}^{\mathrm{b}}=\pi / 4\right)$. (a) Order and (b) velocity distribution with activity number $\alpha=$ $H \sqrt{\zeta / K}=15$. (c) Order and (d) velocity distribution with activity number $\alpha=25.98$. 
the alignment of units [Figs. 7(a) and 7(b)]. Such antiparallel shear flow has been observed in elongated retinal pigment epithelial cells and $\mathrm{C} 2 \mathrm{C} 12$ mouse myoblasts within confining stripes [52]. With increasing activity, the system develops into a chaotic state, where topological defects excited by active stress are inclined to migrate along boundary walls, exhibiting frequent nucleation and annihilation [Figs. 7(c) and $7(\mathrm{~d})]$.
[1] V. Hakim and P. Silberzan, Rep. Prog. Phys. 80, 076601 (2017).

[2] B. Ladoux and R. M. Mège, Nat. Rev. Mol. Cell Bio. 18, 743 (2017).

[3] X. Trepat and E. Sahai, Nat. Phys. 14, 671 (2018).

[4] Y. Sumino et al., Nature (London) 483, 448 (2012).

[5] T. Sanchez, D. T. Chen, S. J. DeCamp, M. Heymann, and Z. Dogic, Nature. 491, 431 (2012).

[6] G. Duclos et al., Science 367, 1120 (2020).

[7] L. Huber, R. Suzuki, T. Krüger, E. Frey, and A. Bausch, Science. 361, 255 (2018).

[8] H. H. Wensink, J. Dunkel, S. Heidenreich, K. Drescher, R. E. Goldstein, H. Löwen, and J. M. Yeomans, Proc. Natl. Acad. Sci. U. S. A. 109, 14308 (2012).

[9] M. M. Genkin, A. Sokolov, O. D. Lavrentovich, and I. S. Aranson, Phys. Rev. X 7, 011029 (2017).

[10] C. Chen, S. Liu, X. Q. Shi, H. Chatè, and Y. L. Wu, Nature (London) 542, 210 (2017).

[11] S. Zhou, A. Sokolov, O. D. Lavrentovich, and I. S. Aranson, Proc. Natl. Acad. Sci. U. S. A. 111, 1265 (2014).

[12] J. Dunkel, S. Heidenreich, K. Drescher, H. H. Wensink, M. Bär, and R. E. Goldstein, Phys. Rev. Lett. 110, 228102 (2013).

[13] J. Toner and Y. H. Tu, Phys. Rev. E 58, 4828 (1998).

[14] T. Vicsek and A. Zafeiris, Phys. Rep. 517, 71 (2012).

[15] O. Peleg, J. M. Peters, M. K. Salcedo, and L. Mahadevan, Nat. Phys. 14, 1193 (2018).

[16] A. Deblais, T. Barois, T. Guerin, P. H. Delville, R. Vaudaine, J. S. Lintuvuori, J. F. Boudet, J. C. Baret, and H. Kellay, Phys. Rev. Lett. 120, 188002 (2018).

[17] D. Bi, X. Yang, M. C. Marchetti, and M. L. Manning, Phys. Rev. X 6, 021011 (2016).

[18] S. Z. Lin, S. Ye, G. K. Xu, B. Li, and X. Q. Feng, Biophys. J. 115, 1826 (2018).

[19] A. Maitra, M. Lenz, and R. Voituriez, Phys. Rev. Lett. 125, 238005 (2020).

[20] V. Schaller and A. R. Bausch, Proc. Natl. Acad. Sci. U. S. A. 110, 4488 (2013).

[21] S. Z. Lin, B. Li, G. Lan, and X. Q. Feng, Proc. Natl. Acad. Sci. U. S. A. 114, 8157 (2017).

[22] J. A. Park et al., Nat. Mater. 14, 1040 (2015).

[23] S. Garcia, E. Hannezo, J. Elgeti, J. F. Joanny, P. Silberzan, and N. S. Gov, Proc. Natl. Acad. Sci. U. S. A. 112, 15314 (2015).

[24] Y. L. Han et al., Nat. Phys. 16, 101 (2020).

[25] D. Boocock, N. Hino, N. Ruzickova, T. Hirashima, and E. Hannezo, Nat. Phys. 17, 267 (2021).

[26] C. Bechinger, R. Di Leonardo, H. Löwen, C. Reichhardt, G. Volpe, and G. Volpe, Rev. Mod. Phys. 88, 045006 (2016).

[27] S. Ramaswamy, Annu. Rev. Condens. Matter Phys. 1, 323 (2010).

[28] M. C. Marchetti, J. F. Joanny, S. Ramaswamy, T. B. Liverpool, J. Prost, M. Rao, and R. A. Simha, Rev. Mod. Phys. 85, 1143 (2013).

[29] H. Chatè, Annu. Rev. Condens. Matter Phys. 11, 189 (2020).
[30] L. Giomi, M. J. Bowick, X. Ma, and M. C. Marchetti, Phys. Rev. Lett. 110, 228101 (2013).

[31] S. Shankar, S. Ramaswamy, M. C. Marchetti, and M. J. Bowick, Phys. Rev. Lett. 121, 108002 (2018).

[32] A. Maitra and M. Lenz, Nat. Commun. 10, 920 (2019).

[33] K. Copenhagen, R. Alert, N. S. Wingreen, and J. W. Shaevitz, Nat. Phys. 17, 211 (2021).

[34] T. B. Saw et al., Nature (London) 544, 212 (2017).

[35] Y. Maroudas-Sacks, L. Garion, L. Shani-Zerbib, A. Livshits, E. Braun, and K. Keren, Nat. Phys. 17, 251 (2021).

[36] D. Needleman and Z. Dogic, Nat. Rev. Mater. 2, 17048 (2017).

[37] A. Doostmohammadi, M. F. Adamer, S. P. Thampi, and J. M. Yeomans, Nat. Commun. 7, 10557 (2016).

[38] S. P. Thampi, R. Golestanian, and J. M. Yeomans, Phys. Rev. E 90, 062307 (2014).

[39] G. Duclos, C. Erlenkämper, J. F. Joanny, and P. Silberzan, Nat. Phys. 13, 58 (2017).

[40] K. Thijssen, M. R. Nejad, and J. M. Yeomans, Phys. Rev. Lett. 125, 218004 (2020).

[41] Z. Y. Li, D. Q. Zhang, S. Z. Lin, and B. Li, Phys. Rev. Lett. 125, 098002 (2020).

[42] L. A. Hoffmann, K. Schakenraad, R. M. H. Merks, and L. Giomi, Soft Matter 16, 764 (2020).

[43] T. N. Shendruk, A. Doostmohammadi, K. Thijssen, and J. M. Yeomans, Soft Matter 13, 3853 (2017).

[44] A. Doostmohammadi, J. Ignés-Mullol, J. M. Yeomans, and F. Sagués, Nat. Commun. 9, 3246 (2018).

[45] S. R. K. Vedula, M. C. Leong, T. L. Lai, P. Hersen, A. J. Kabla, C. T. Lim, and B. Ladoux, Proc. Natl. Acad. Sci. U. S. A. 109, 12974 (2012).

[46] A. Opathalage, M. M. Norton, M. P. N. Juniper, B. Langeslay, S. A. Aghvami, S. Fraden, and Z. Dogic, Proc. Natl. Acad. Sci. U. S. A. 116, 4788 (2019).

[47] C. Blanch-Mercader, P. Guillamat, A. Roux, and K. Kruse, Phys. Rev. Lett. 126, 028101 (2021).

[48] A. Doostmohammadi, T. N. Shendruk, K. Thijssen, and J. M. Yeomans, Nat. Commun. 8, 15326 (2017).

[49] J. Hardoüin, R. Hughes, A. Doostmohammadi, J. Laurent, T. Lopez-Leon, J. M. Yeomans, J. Ignés-Mullol, and F. Sagués, Commun. Phys. 2, 121 (2019).

[50] S. Chandragiri, A. Doostmohammadi, J. M. Yeomans, and S. P. Thampi, Phys. Rev. Lett. 125, 148002 (2020).

[51] M. Varghese, A. Baskaran, M. F. Hagan, and A. Baskaran, Phys. Rev. Lett. 125, 268003 (2020).

[52] G. Duclos, C. Blanch-Mercader, V. Yashunsky, G. Salbreux, J. F. Joanny, J. Prost, and P. Silberzan, Nat. Phys. 14, 728 (2018).

[53] L. Yamauchi, T. Hayata, M. Uwamichi, T. Ozaway, and K. Kawaguchi, arXiv:2008.10852 (2020).

[54] J. Elgeti and G. Gompper, Proc. Natl. Acad. Sci. U. S. A. 110, 4470 (2013). 
[55] M. L. Blow, M. Aqil, B. Liebchen, and D. Marenduzzo, Soft Matter 13, 6137 (2017).

[56] L. Giomi, Phys. Rev. X 5, 031003 (2015).

[57] E. J. Hemingway, P. Mishra, M. C. Marchetti, and S. M. Fielding, Soft Matter 12, 7943 (2016).

[58] A. N. Beris and B. J. Edwards, Thermodynamics of Flowing Systems: With Internal Microstructure (Oxford University Press, Oxford, 1994).

[59] P. G. de Gennes and J. Prost, The Physics of Liquid Crystals (Oxford University Press, Oxford, 1993).

[60] S. Čopar, J. Aplinc, Ž. Kos, S. Žumer, and M. Ravnik, Phys. Rev. X 9, 031051 (2019).

[61] R. Zhang, Y. Zhou, M. Rahimi, and J. J. de Pablo, Nat. Commun. 7, 13483 (2016).

[62] A. Sokolov, A. Mozaffari, R. Zhang, J. J. de Pablo, and A. Snezhko, Phys. Rev. X 9, 031014 (2019).

[63] E. Orlandini, M. E. Cates, D. Marenduzzo, L. Tubiana, and J. M. Yeomans, Mol. Cryst. Liq. Cryst. 494, 293 (2008).

[64] L. Giomi, M. J. Bowick, P. Mishra, R. Sknepnek, and M. C. Marchetti, Phil. Tran. R. Soc. A 372, 20130365 (2014).

[65] J. Prost, F. Jülicher, and J. F. Joanny, Nat. Phys. 11, 111 (2015).

[66] F. Jülicher, S. W. Grill, and G. Salbreux, Rep. Prog. Phys. 81, 076601 (2018).

[67] R. Green, J. Toner, and V. Vitelli, Phys. Rev. Fluids 2, 104201 (2017).
[68] S. P. Thampi, R. Golestanian, and J. M. Yeomans, Europhys. Lett. 105, 18001 (2014).

[69] R. Voituriez, J. F. Joanny, and J. Prost, Europhys. Lett. 70, 404 (2005).

[70] J. Toner and Y. Tu, Phys. Rev. Lett. 75, 4326 (1995).

[71] S. Shankar, M. J. Bowick, and M. C. Marchetti, Phys. Rev. X 7, 031039 (2017).

[72] K. Sone and Y. Ashida, Phys. Rev. Lett. 123, 205502 (2019).

[73] A. Souslov, B. C. van Zuiden, D. Bartolo, and V. Vitelli, Nat. Phys. 13, 1091 (2017).

[74] N. Manton and P. Sutcliffe, Topological Solitons (Cambridge University Press, Cambridge, 2007).

[75] K. Thijssen and A. Doostmohammadi, Phys. Rev. Research 2, 042008(R) (2020).

[76] Y. F. Zhang, B. Li, Q. S. Zheng, G. M. Genin, and C. Q. Chen, Nat. Commun. 10, 5605 (2019).

[77] B. L. Deng, S. Q. Yu, A. E. Forte, V. Tournat, and K. Bertoldi, Proc. Natl. Acad. Sci. U. S. A. 117, 31002 (2020).

[78] P. Marin-Palomo et al., Nature (London) 546, 274 (2017).

[79] R. Zhang, A. Mozaffari, and J. de Pablo, Nat. Rev. Mater. 6, 437 (2021).

[80] C. Peng, T. Turiv, Y. Guo, Q. H. Wei, and O. D. Lavrentovich, Science 354, 882 (2016).

[81] T. Turiv et al., Sci. Adv. 6, eaaz6485 (2020). 